\title{
Capability of an Ultrasonic System to Detect Very Early Caries Lesions on Human Enamel
}

\author{
Funda Öztürk Bozkurt ${ }^{1}$, Dilek Tağtekin ${ }^{2}$, Funda Yanıkoğlu², Margherita Fontana ${ }^{3}$, \\ Carlos Gonzalez-Cabezas, George K.Stookey ${ }^{4}$
}

\begin{abstract}
The purpose of this investigation was to determine if changes in dental enamel with 20 micron depth incipient carious lesion could be detected by an ultrasonic system (US). Natural (unground, unpolished) lesions were produced on human enamel by using a microbial caries model. Specimens with lesions were analyzed using Ultrasonic system (US) as test method, Quantitative-Light Induced Fluorescence (QLF) and Confocal Laser Scanning Microscopy (CLSM) as gold standards. It was found that both ultrasound and QLF could not detect these very early lesions (mean lesion depth: $18.89 \mu \mathrm{m})$ created in vitro in the microbial caries model.
\end{abstract}

Keywords: Ultrasound, Microhardness, QLF, Confocal Microscope, PreWhite Spot Caries Lesion.

\section{Introduction}

In the industrial world subsurface defects are easily identified when an ultrasonic beam has been directed perpendicular to the surface. Because the time of flight (travel time) of the sound waves will be different in two different mediums it is possible to differentiate between sound and demineralized enamel (1). Sonic waves that are reflected back (echoes) toward the transducer cause a change in the thickness of the piezoelectric crystal, which in turn produces an electrical signal that can be amplified, processed and ultimately stored (2).

Ultrasound imaging in dentistry has been employed in some laboratory studies focusing on the dentino-enamel junction, oral soft

\footnotetext{
${ }^{1}$ Department of Restorative Dentistry, Faculty of Dentistry, University of Medipol, Istanbul,Türkiye

${ }^{2}$ Department of Restorative Dentistry, Faculty of Dentistry, University of Marmara Istanbul,Türkiye

${ }^{3}$ Department of Cariology, Restorative Sciences and Endodontics,

School of Dentistry, University of Michigan, Ann Arbor, Michigan, USA

${ }^{4}$ Therametric Technologies, Inc., Noblesville, Indiana, USA

Correspondence Address to:

Dilek Tağtekin

Marmara University Faculty of Dentistry

Buyukciftlik Sok. No: 6, 34365, Nisantasi, Istanbul, Türkiye

Tel : : +902122319120

Fax : :90212 2465247

E-mail: dtagtekin@marmara.edu.tr
}

tissues and skeletal defects (3-9). Nondestructive ultrasound system was found amazing by some dental researchers (10). Few authors have worked on demineralization on enamel (11-15). The systems they used were not consistent and practical and the coupling agents were made of various materials, therefore different results were obtained. Fontana et al. used the ultrasonic systems on detection of artificial carious lesions (16). Although they used different systems, all agreed on its effectiveness on differentiation of small carious enamel lesions. The most recent study on this subject revealed that ultrasound could differentiate the densitometric changes of human enamel with natural carious lesion with numerical values (17).

The hypothesis tested in this investigation was that the ultrasonic system could be used for the detection of very early dental caries lesion. Therefore, the study was planned to evaluate an industrial ultrasonic system for detection of a very early caries lesion with $20 \mu \mathrm{m}$ in depth produced by microbial caries model and assessed with QLF and confocal microscope methods.

\section{Materials and Methods}

\section{Specimen Preparation:}

Sixty-four enamel specimens (3 $\mathrm{mm}$ in diameter) were obtained from extracted, human maxillary premolars and stored in $10 \%$ buffered formalin ( $\mathrm{pH}$ 6.8-7.0). Each specimen was mounted on a polyacrylic rod using denture acrylic and was randomly coded with a two-digit number. Specimens were not ground or, polished and, therefore, retained their natural surface.

\section{Baseline Quantitative Light-Induced Fluorescence Analysis (QLF I):}

Before lesion formation, all specimens were analyzed using the QLF system (QLF/clin 007, Inspektor Research Systems B.V., The Netherlands) to obtain baseline images. This system consists of a lamp unit and a camera control unit, which is connected via a liquid light guide and electrical cable to a camera handpiece. Specimens were exposed to $13 \mathrm{mw} / \mathrm{cm} 2$ of violet-blue light (wave length: 290$450 \mathrm{~nm}$ ) via the liquid light guide in the camera handpiece. One image was captured through a $510 \mathrm{~nm}$ band-pass filter, using a miniature CCD camera located inside the handpiece. The images were stored in the QLF 1.96w program. 


\section{Baseline Ultrasonic Analysis:}

The ultrasonic system was composed of a transducer (probe) (Harisonic, Staveley, NDT, WA, USA) and an ultrasonic precision thickness gauge (NDT, Novascope 4500, MA, USA). The contact transducer was of the right angle-type, with a $1.5 \mathrm{~mm}$ tip contact diameter, $11 \mathrm{MHz}$ nominal center frequency, and with a removable plexiglass delay tip. The frequency range of the system was between $0.3-20 \mathrm{MHz}$ and the pulse rate was 50 to $3450 \mathrm{~Hz}$. The ultrasonic machine chooses automatically the pulse rate depending on the selected time base range. The thickness reading of the instrument was used as meters with a precision of one promille (1/1000 inches).

All ultrasonic testing done at the NDT Division of Turkish Airlines, Istanbul, Türkiye. The specimens were dried for five seconds using an air blast. Glycerin (Fisher Scientific) was used as a coupling medium between the probe and the specimen surfaces. The ultrasonic test equipment with the transducer (probe) was calibrated each time according to a standard calibration procedure (at ambient temperature) on titanium calibration blocks (Combuster cowl thickness calibration standard CT 3392 S 7N 96-027, Novascope 4500). The calibration was made at 0.025 and 0.035 inches in depth at each measurement of each surface $(5979 \mathrm{~m} / \mathrm{sec})$. A 1.5 - $\mathrm{mm}$ thick enamel section was cut from the buccal surface of an intact tooth and used for calibration at the beginning and end of the test procedures with $6132 \mathrm{~m} / \mathrm{sec}$ velocity and $1.5 \mathrm{~mm}$ in thickness.

The probe was oriented perpendicular to the surface during the contact with each specimen to get the echo signals as calibrated before. When an echo amplitude was obtained on the CRT (Oscilloscope peaks) that was lower than the control value for the specimen, it was accepted as presence of a lesion and its numeric value stored. The measurements were repeated three times for each specimen with five seconds pause between each measurement. The nominal values gave the information about changes of tissue in the lesion areas. Two examiners were trained by the staff that routinely uses the ultrasonic machine. The obtained surface echo values were evaluated by statistical analysis.

\section{Lesion Formation:}

Fifty-six specimens were demineralized using an in vitro microbial caries model [18] and eight specimens served as non demineralizedbaseline controls. There were four identical groups, and each contained 14 enamel specimens. The specimens were gas sterilized before being inoculated and placed in the artificial mouth model. The baseline control specimens were also gas sterilized, but were not placed in the artificial mouth model.

All groups were inoculated once at the beginning of the experiment with $1.2 \times 108 \mathrm{CFU} / \mathrm{ml}$ of Streptococcus mutans A32-2 (serotype c). Specimens were exposed to circulating trypticase soy broth supplemented with $5 \%$ sucrose (TSBS) and mineral washing solution (MW), for 30 minutes, 3 times a day, and a total of 22.5 hours per day, respectively. At the end of the study, three teeth/group were aseptically removed, placed in $5 \mathrm{ml}$ of sterile saline, and vortexed $(20 \mathrm{sec})$ and sonicated $(20 \mathrm{sec})$ to disrupt plaque from the tooth surface. The dislodged bacteria were then double plated, undiluted and diluted 1:1000 and 1:10000, in Mitis Salivarus supplemented with bacitracin and sucrose (MSSB) and Trypticase Soy Agar (TSA) for bacterial quantitation and to check for lack of contamination, respectively.

\section{Demineralization QLF Analysis (QLF II):}

Following lesion development, specimens were cleaned in deionized water and analyzed using the QLF system to obtain post-demineralization images. In order to calculate fluorescence loss in the demineralized enamel, the sound enamel fluorescence radiance at the demineralized enamel surface was reconstructed by the interpolation of sound enamel fluorescence radiance (obtained from the baseline image). Briefly, QLF images of all specimens were modified using Adobe Photoshop software (version 4.0.1; Adobe Systems Inc., WA, USA). A part of the demineralized enamel image (QLF II) was cut and pasted in the center of the same specimen's sound enamel image (QLF I). In order to calculate the fluorescence loss in the demineralized area using the QLF program, a computer-generated rectangle was placed in the area of analysis. This computer-generated rectangle defining the region of interest was manipulated so that the edges of the rectangle lay over sound enamel. The size of the rectangle was kept constant for all the analyses. The fluorescence values of the pixels within the rectangle were compared to the fluorescence values of the pixels surrounding the rectangle (sound tissue). The sound enamel fluorescence radiance inside the rectangle was reconstructed from the gray-pixel values at the rectangle borders. Lesion threshold was set at $95 \%$, indicating that fluorescence values at less than $95 \%$ of their reference fluorescence values were considered demineralized. The difference between original pixel values and the reconstructed sound pixel values of areas with fluorescence levels below the threshold of $95 \%$ gave the resulting fluorescence loss in the demineralized enamel. This difference was recorded as \%average change in fluorescence and $\Delta \mathrm{Q}(\Delta \mathrm{Q}=$ \%average change in fluorescence $\mathrm{x}$ area in $\mathrm{mm} 2)$.

\section{Demineralization Ultrasonic Analysis:}

The specimens were dried for five seconds using an air blast and analyzed with the ultrasonic system (NDT, Novascope, 4500, Waltham, MA, USA) for detection of pulsed-longitudinal wave echo signals from the surfaces as the same manner as for the baseline ultrasonic analysis.

\section{Confocal Laser Scanning Microscopy (CLSM) Analysis:}

Specimens were sectioned in half using a water-cooled, SilverstoneTaylor hard tissue microtome (Scientific Fabrications Laboratory, Lafayette, CO, USA). One half of each tooth was stained overnight with a freshly prepared $0.1 \mathrm{mM}$ Rhodamine B solution (Aldrich Chem. Co., Milwaukee, Wisc., USA), without further rinsing. For each specimen, measurements were made in one $340 \mu \mathrm{m}$ in length area and analyzed for depth, area, and total fluorescence as previously described [19]. Briefly, the cut, stained surface of each specimen was allowed to air dry before being analyzed with the confocal laser scanning microscope (Odyssey, Noran Instruments, Inc., Middleton, Wisc., USA). Images were analyzed with the Image 1 (version 4.14.C) software (Universal Images Corp., West Chester, PA, USA). After being brought into focus (using a 10X Nikon objective, N.A. 0.25 ), the specimens were illuminated with an argon ion laser using a 488-nm excitation wavelength. Confocal slits were set at $15 \mathrm{~mm}$ with a $515 \mathrm{~nm}$ long-pass barrier filter. For collection of the images, samples were frame-averaged using 128 frames per image. Areas were scanned planoparallel to the transversal cut surface of the specimen and perpendicular to the natural surface of the tooth. 


\section{Statistical Analysis:}

Data were analyzed using a single factor analysis of variance model (ANOVA), and if a significant $\mathrm{F}$ value was found $(\mathrm{p}<0.05)$, multiple comparisons were conducted using Tukey's procedure.

\section{Results}

\section{In vitro Microbial Model:}

The $\mathrm{pH}$ of the fluid in the drainage containers and caries vessels was monitored at the end of the demineralization experimental phase in order to document the acidic conditions created by the bacterial inoculum (4.43-4.59). There were no differences in the final $\mathrm{pH}$ of the 4 vessels. This suggested the acidic challenge had been similar in all groups.

At the end of the treatment period plaque was harvested from 3 treated specimens in each of the groups and was monitored for bacterial counts. Drainage container fluid was also plated to monitor for bacteria viability and lack of contamination. There was no significant difference among the 4 groups ( $\mathrm{p}>0.05$ ).

\section{Comparison of all 3 techniques to sound baselines:}

Since all 4 groups in the microbial model were similar, all 56 specimen's data were combined for analysis. The ability of the techniques to distinguish between sound and demineralized natural enamel a surface was studied (Table 1). The data indicate that CLSM could detect demineralization following the in vitro microbial caries model challenge (mean lesion depth: $18.89 \mu \mathrm{m}$ ) compared to sound baselines; however, neither ultrasound nor QLF could not detect these lesions from the surrounding background.

Table 1. QLF, Ultrasonic and CLSM measurements of Primary Carious Lesions following demineralization.

\begin{tabular}{|c|c|c|c|c|}
\hline $\begin{array}{l}\text { Type of } \\
\text { Specimen }\end{array}$ & $\begin{array}{c}\text { CLSM } \\
\text { (Depth) } \\
(\mu \mathrm{m}) \\
\text { Mean } \pm \text { SD }\end{array}$ & $\begin{array}{c}\text { Ultrasound } \\
\text { (1. researcher) } \\
(\mathrm{mm}) \\
\text { Mean } \pm \text { SD }\end{array}$ & $\begin{array}{c}\text { Ultrasound } \\
(2 . \\
\text { researcher }) \\
(\mathrm{mm}) \\
\text { Mean } \pm \text { SD } \\
\end{array}$ & $\begin{array}{c}\text { QLF } \\
(\Delta \mathbf{Q}) \\
\text { Mean } \pm \text { SD }\end{array}$ \\
\hline $\begin{array}{l}\text { Sound } \\
\text { (Baseline) }\end{array}$ & $0+0$ & $\begin{array}{c}1162 \pm \\
154\end{array}$ & $\begin{array}{c}1134 \pm \\
91\end{array}$ & $\begin{array}{c}-13.8 \pm \\
5.1\end{array}$ \\
\hline Demineralized & $\begin{array}{c}18.89 \pm \\
17.26\end{array}$ & $\begin{array}{c}1088 \pm \\
140\end{array}$ & $\begin{array}{c}1186 \pm \\
198\end{array}$ & $\begin{array}{c}-16.6 \pm \\
4.8\end{array}$ \\
\hline
\end{tabular}

Groups within brackets were not significantly different $(\mathrm{p}>0.05)$.

\section{Discussion}

Our previous study had suggested that the ultrasonic system can be sensitive enough to detect differences in enamel tissue density (17). Also, specific acoustic impedance of mineral content of the body of the enamel lesion had been reported in the literature (15). They produced artificial lesions in 6\% hydroxyethylcellulose gel in a period of 1, 3 to 7 days, at $\mathrm{pH} 4.4$ and a temperature of $200 \mathrm{C}$, on $1.5 \times 1.5 \mathrm{~mm}$ size windows on the labial surfaces of six extracted human permanent anterior teeth. Lesions varying from 116-168 micron thickness were detected by the ultrasonic system they used.

Çalışkan et. al. (17) reported the ultrasonic system appeared to be sensitive and be obtained numeric values resulting from differences in tissue density. Approximate lesion depth was around 70 micrometer. The study by Fontana et al. reported the in vitro microbial caries model can produced uniform, very small lesions (mean: $18.27+$ $2.89 \mu \mathrm{m}$ in depth) in natural surface enamel specimens. Preliminary studies had demonstrated that CLSM, the gold standard, could accurately detect early lesions of approximately 20 microns in depth (19). These lesions were non-detectable (pre-white spot) with visual examination and in this study both ultrasonic detection and QLF were not able to detect those lesions either. An explanation for the lack of detection of caries by ultrasound could be that the lesions studied were beyond the limit of detection for the instrument. Even though the studies by Kwon and Katz [9]; Katz and Akraincik (20) showed that ultrasonics could detect very small changes in crystal structures causing change in density. This leads to a change in acoustic velocity measurements that can be detected and displayed in a milimetric box. The lesions in the present study were probably so superficial that the ultrasonic system was not be able to differentiate from natural surface.

This study confirmed that, only CLSM was able to detect the initially demineralized specimens from the sound controls, while the ultrasonic technique and QLF in 20 micron depth could not detect this very superficial lesions.

It is concluded that the ultrasonic system used in this investigation was not able to detect $20 \mu \mathrm{m}$ very early caries lesions. Further studies are required to establish the threshold of the system on detection of very early enamel lesions.

\section{Acknowledgments}

We are very grateful to engineers Fikret Geçgil and Ercan Kocagil and the NDT Team of Turkish Airlines for their help and the instructions about the ultrasonic system.

\section{References}

1. Richter HU. Verfahren der Ultraschall-Materialprüfung. Materialprüf 1974; 308:10;10-16.

2. Krautkramer J, Krautkramer H. Ultrasonic testing of materials. 2nd ed. New York, Springer-Verlag, 1977.

3. Baum G, Greenwood I, Slawski S et al. Observation of internal structures of teeth by ultrasonography. Science 1963;139:495-496.

4. Lees S, Barber FE. Looking into teeth with ultrasound. Science 1968;161:477-478.

5. Barber FE, Lees S, and Lobene RR. Ultrasonic pulse-echo measurements in teeth. Arch Oral Biol 1969;14:745-760.

6. Daly CH, Wheeler JB. The use of ultrasonic thickness measurement in the clinical evaluation of the oral soft tissues. Int Dent J 1971; 21:418429.

7. Spranger $\mathrm{H}$. Ultrasonic diagnosis of marginal periodontal diseases. Int Dent J 1971; 21:442-455.

8. Fukukita H, Yano T, Fukumoto A et al. Development and application of an ultrasonic imaging system for dental diagnosis. J Clin Ultrasound 1985;13:597-600. 
9. Kwon S, Katz JL. Sonic diagnosis of skeletal defects: a preliminary study. Adv Dent Res 1987;1:39-44.

10. Scheven BAA: Therapeutic ultrasound for dental tissue repair. Medical Hypotheses 2009;73: 591-593.

11. Lees S, Barber FE, Lobene RR. Dental enamel: detection of surface changes by ultrasound. Science 1970;169:1314-1316.

12. Lees $\mathrm{S}$, and Barber FE. Looking into the tooth and its surfaces with ultrasonics. Ultrasonics 1971;9:95-100.

13. Lees S, Gerhard FB, Oppenheim FG. Ultrasonic measurement of dental enamel demineralization. Ultrasonics 1973;11:269-273.

14. Peck SD, Briggs GAD. A scanning acoustic microscope study of the small caries lesion in human enamel. Caries Res 1986;20:356-360.

15. Ng SY, Ferguson MWJ, Payne PA, Slater P: Ultrasonic studies of unblemished and artificially demineralized enamel in extracted human teeth: a new method for detecting early caries. J Dent 1988;16: 201-209.

16. Fontana M., Çalışkan Yanıkoğlu F, Öztürk F, Ando M, Hayran O., Gonzalez-Cabezas C., Haider A, Baldridge LA, Stookey GK.: Comparison of QLF, Ultrasound and Confocal Microscopy in the measurement of Demineralization/Remineralization of Enamel Lesions Developed on Natural Smooth Surfaces. Early Detection of Dental Caries 11, Indiana Conference, Indiana, 19-22 May, 1999.

17. Çalışkan FY, Öztürk F, Hayran O, Analoui M, Stookey GK. Detection of natural white spot caries lesions by an ultrasonic system. Caries Res 2000;34:225-232.

18. Fontana M, Dunipace AJ, Gregory RL, Noblitt TW, Li Y, Park KK. An in-vitro microbial model for studying secondary caries formation. Caries Res 1996 ;30:112-118.

19. Fontana M, Li Y, Dunipace AJ, Noblitt TW, Fischer GM, Katz BP. Measurement of demineralization of enamel using microradiography and confocal microscopy. A correlational study. Caries Res 1996;30:317-325.

20. Katz JL, Akraincik K. On the anisotropic properties of hydroxyapatite. J Biomechanics 1971;4:221-227. 